The Discovery of Dihydropyrrolo[1,2-a]pyrazin-3(4H)-one-Based Second-Generation

GluN2C- and GluN2D-Selective Positive Allosteric Modulators (PAMs) of the $N$-Methyl-DAspartate (NMDA) Receptor

Matthew P. Epplin, ${ }^{\dagger}$ Ayush Mohan,${ }^{\dagger}$ Lynnea D. Harris,${ }^{\dagger}$ Zongjian Zhu,,${ }^{\ddagger}$ Katie L. Strong,,${ }^{\dagger}$ John

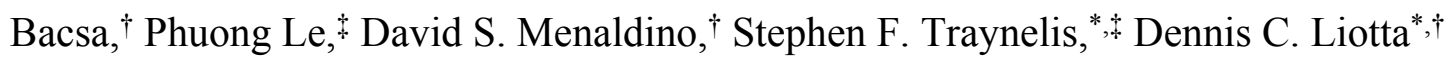

$\dagger^{\dagger}$ Emory University, Department of Chemistry, 1515 Dickey Drive, Atlanta, GA 30322, USA Emory University, Department of Pharmacology and Chemical Biology, 1510 Clifton Road, Atlanta, GA 30322, USA *Co-corresponding Authors

Table of Contents

\begin{tabular}{|c|c|}
\hline Page & Supplemental Data \\
\hline S2 & Supplemental Table S1 \\
\hline S3 & Supplemental Table S2 \\
\hline S4 & Supplemental Table S3 \\
\hline S5-6 & Supplemental Table S4 \\
\hline S7 & Supplemental Table S5 \\
\hline S8-9 & Supplemental Table S6 \\
\hline S10 & Crystal Structure Methods \\
\hline S11-13 & Chemistry Experimental Details for Compound 36 \\
\hline S14-20 & Chemistry Experimental Details for S1-S6 \\
\hline S21 & References \\
\hline
\end{tabular}


Supplemental Table S1. Potentiation of GluN2A- and GluN2B-Containing Receptors

\begin{tabular}{|c|c|c|}
\hline$\#$ & $\begin{array}{c}\text { GluN1/GluN2A } \boldsymbol{I}_{10 \mu M} / \boldsymbol{I}_{\text {control }}{ }^{a} \\
(\operatorname{mean} \pm \mathbf{S E M}, \%)\end{array}$ & $\begin{array}{c}\text { GluN1/GluN2B } \boldsymbol{I}_{10 \mu M} / \boldsymbol{I}_{\text {control }}{ }^{a} \\
(\text { mean } \pm \text { SEM, } \%)\end{array}$ \\
\hline 12d & $140 \pm 10$ & $190 \pm 10$ \\
\hline $12 \mathrm{c}$ & $110 \pm 0^{b}$ & $130 \pm 10$ \\
\hline
\end{tabular}

${ }^{a}$ The ratio of the current response to $100 \mu \mathrm{M}$ glutamate and $30 \mu \mathrm{M}$ glycine with test compound to the response to glutamate and glycine alone is shown for oocytes expressing either GluN1/GluN2A- or GluN1/GluN2B-containing receptors. Data are from 7-13 oocytes from 2-3 frogs. ${ }^{b}$ Current ratio reported for $30 \mu \mathrm{M}$. 
Supplemental Table S2. Crystal Data and Structure Refinement for $R$-(+)-EU-1180-453

\begin{tabular}{|c|c|}
\hline Formula & $\mathrm{C}_{22} \mathrm{H}_{21} \mathrm{FN}_{2} \mathrm{O}_{3}$ \\
\hline$D_{\text {calc. }} / \mathrm{g} \mathrm{cm}^{-3}$ & 1.359 \\
\hline$\mu / \mathrm{mm}^{-1}$ & 0.806 \\
\hline Formula Weight & 380.41 \\
\hline Colour & colorless \\
\hline Shape & block \\
\hline Size $/ \mathrm{mm}^{3}$ & $0.40 \times 0.32 \times 0.22$ \\
\hline$T / \mathrm{K}$ & 99.99(2) \\
\hline Crystal System & monoclinic \\
\hline Flack Parameter & $-0.1(2)$ \\
\hline Hooft Parameter & $-0.01(10)$ \\
\hline Space Group & $P 2_{1}$ \\
\hline$a / \AA$ & $9.5577(5)$ \\
\hline$b / \AA$ & $6.4506(3)$ \\
\hline$c / \AA$ & $15.2496(8)$ \\
\hline$\alpha /^{\circ}$ & 90 \\
\hline$\beta /^{\circ}$ & $98.508(5)$ \\
\hline$\gamma^{\circ}$ & 90 \\
\hline$V / \AA^{3}$ & $929.84(8)$ \\
\hline Z & 2 \\
\hline$Z^{\prime}$ & 1 \\
\hline Wavelength $/ \AA$ & 1.54184 \\
\hline Radiation type & $\mathrm{CuK}_{\alpha}$ \\
\hline$\Theta_{\min } /{ }^{\circ}$ & 4.678 \\
\hline$\Theta_{\max } /{ }^{\circ}$ & 68.243 \\
\hline Measured Refl. & 9222 \\
\hline Independent Refl. & 2879 \\
\hline Reflections with $\mathrm{I}>2 \sigma(\mathrm{I})$ & 2757 \\
\hline$R_{\text {int }}$ & 0.0665 \\
\hline Parameters & 292 \\
\hline Restraints & 84 \\
\hline Largest Peak & 0.233 \\
\hline Deepest Hole & -0.219 \\
\hline GooF & 1.042 \\
\hline$w R_{2}$ (all data) & 0.1166 \\
\hline$w R_{2}$ & 0.1147 \\
\hline$R_{I}$ (all data) & 0.0457 \\
\hline$R_{1}$ & 0.0439 \\
\hline
\end{tabular}


Supplemental Table S3. Potentiation of GluN2A-D Upon Co-Application of Compound $12 \mathrm{~m}$ and Sub-Saturating Concentrations of Glutamate and Glycine

\begin{tabular}{|c|c|c|c|c|}
\hline \multirow[b]{2}{*}{ \# } & \multicolumn{4}{|c|}{$\begin{array}{c}I_{10 \mu \mathrm{M}} / I_{\text {control }}(\operatorname{mean} \pm \mathrm{SEM}, \%) \\
{[\text { Low Glutamate Concentration / Low Glycine Concentration, } \mu \mathrm{M}]^{a}}\end{array}$} \\
\hline & GluN2A & GluN2B & GluN2C & GluN2D \\
\hline $12 m$ & $96 \pm 5[1.9 / 0.8]$ & $88 \pm 4[0.8 / 0.5]$ & $450 \pm 10[0.9 / 0.2]$ & $400 \pm 30[0.3 / 0.1]$ \\
\hline
\end{tabular}

${ }^{a}$ The current ratio in presence of $10 \mu \mathrm{M}$ drug to control is give as $\%$. Glutamate and glycine concentrations are given in brackets. Data are from between 4 and 8 oocytes from 1 frog for each compound and receptor tested. 
Supplemental Table S4. Subset of Compounds Demonstrating SAR is Transferable Between TIQ Scaffold and Dihydroisoquinolinone Scaffold<smiles>[R]Oc1ccc(OCC2c3cc([R])c(OC)cc3CC([Y])N2C([X])c2ccc([R3])c([R])c2[R])cc1</smiles>

\begin{tabular}{|c|c|c|c|c|c|c|c|c|c|c|}
\hline \multirow[b]{2}{*}{ \# } & \multirow[b]{2}{*}{$\mathbf{R}_{1}$} & \multirow[b]{2}{*}{$\mathbf{R}_{2}$} & \multirow[b]{2}{*}{$\mathbf{R}_{3}$} & \multirow[b]{2}{*}{$\mathbf{R}_{4}$} & \multirow[b]{2}{*}{$\mathbf{R}_{\mathbf{5}}$} & \multirow[b]{2}{*}{$\mathbf{X}$} & \multirow[b]{2}{*}{$\mathbf{Y}$} & \multicolumn{2}{|c|}{$\mathrm{pEC}_{50}[95 \% \mathrm{CI}](\max )(\%)^{a}$} & \multirow{2}{*}{$\begin{array}{c}\text { p(doubling } \\
\text { conc.) } \\
\text { (GluN2C/2D) }\end{array}$} \\
\hline & & & & & & & & GluN2C & GluN2D & \\
\hline $\mathbf{C I Q}^{b}$ & $\mathrm{H}$ & $\mathrm{Cl}$ & $\mathrm{H}$ & $\mathrm{OMe}$ & $\mathrm{Me}$ & $\mathrm{O}$ & - & $5.3(230)$ & $5.3(220)$ & $4.9 / 4.5$ \\
\hline $12 \mathrm{e}$ & $\mathrm{H}$ & $\mathrm{Cl}$ & $\mathrm{H}$ & $\mathrm{H}$ & $\mathrm{Me}$ & - & $\mathrm{O}$ & $\begin{array}{c}5.7[5.6-5.8] \\
(270)\end{array}$ & $\begin{array}{c}5.6[5.5-5.8] \\
(330)\end{array}$ & $5.6 / 5.7$ \\
\hline $188^{b}$ & $\mathrm{Cl}$ & $\mathrm{H}$ & $\mathrm{H}$ & $\mathrm{OMe}$ & $\mathrm{Me}$ & $\mathrm{O}$ & - & ND (100) & ND (100) & - \\
\hline S1 & $\mathrm{Cl}$ & $\mathrm{H}$ & $\mathrm{H}$ & $\mathrm{H}$ & $\mathrm{Me}$ & - & $\mathrm{O}$ & $\begin{array}{c}5.2[5.1-5.3] \\
(230)\end{array}$ & $\begin{array}{c}5.0[4.9-5.1] \\
(250)\end{array}$ & $4.7 / 4.7$ \\
\hline $187^{b}$ & $\mathrm{H}$ & $\mathrm{H}$ & $\mathrm{Cl}$ & $\mathrm{OMe}$ & $\mathrm{Me}$ & $\mathrm{O}$ & - & $5.4(140)$ & $\mathrm{ND}(110)$ & - \\
\hline S2 & $\mathrm{H}$ & $\mathrm{H}$ & $\mathrm{Cl}$ & $\mathrm{H}$ & $\mathrm{Me}$ & - & $\mathrm{O}$ & $\begin{array}{c}5.6[5.5-5.7] \\
(280) \\
\end{array}$ & $\begin{array}{c}5.3[5.2-5.4] \\
(360) \\
\end{array}$ & $5.5 / 5.5$ \\
\hline $1^{* b, c}$ & $\mathrm{H}$ & $\mathrm{H}$ & $\mathrm{H}$ & $\mathrm{OMe}$ & $\mathrm{Me}$ & $\mathrm{O}$ & - & $4.9(150)$ & $5.0(160)$ & - \\
\hline $\mathbf{S 3}$ & $\mathrm{H}$ & $\mathrm{H}$ & $\mathrm{H}$ & $\mathrm{H}$ & $\mathrm{Me}$ & - & $\mathrm{O}$ & $\begin{array}{c}5.2[5.0-5.4] \\
(240)\end{array}$ & $\begin{array}{c}5.2[5.1-5.3] \\
(330)\end{array}$ & $4.7 / 5.3$ \\
\hline $198^{b}$ & $\mathrm{H}$ & $\mathrm{CF}_{3}$ & $\mathrm{H}$ & $\mathrm{OMe}$ & $\mathrm{Me}$ & $\mathrm{O}$ & - & $5.6(200)$ & $5.6(220)$ & - \\
\hline S4 & $\mathrm{H}$ & $\mathrm{CF}_{3}$ & $\mathrm{H}$ & $\mathrm{H}$ & $\mathrm{Me}$ & - & $\mathrm{O}$ & $\begin{array}{c}6.0[5.9-6.1] \\
(250) \\
\end{array}$ & $\begin{array}{c}5.9[5.8-6.1] \\
(320) \\
\end{array}$ & $5.7 / 6.0$ \\
\hline $197^{b}$ & $\mathrm{H}$ & $\mathrm{F}$ & $\mathrm{H}$ & $\mathrm{OMe}$ & $\mathrm{Me}$ & $\mathrm{O}$ & - & $5.1(180)$ & $5.2(170)$ & - \\
\hline S5 & $\mathrm{H}$ & $\mathrm{F}$ & $\mathrm{H}$ & $\mathrm{H}$ & $\mathrm{Me}$ & - & $\mathrm{O}$ & $\begin{array}{c}5.4[5.3-5.4] \\
(350)\end{array}$ & $\begin{array}{c}5.4[5.3-5.5] \\
(380)\end{array}$ & $5.5 / 5.6$ \\
\hline $100^{b}$ & $\mathrm{H}$ & $\mathrm{Cl}$ & $\mathrm{H}$ & $\mathrm{OMe}$ & Et & $\mathrm{O}$ & - & ND (93) & ND (92) & - \\
\hline S6 & $\mathrm{H}$ & $\mathrm{F}$ & $\mathrm{H}$ & $\mathrm{H}$ & Et & - & $\mathrm{O}$ & $\mathrm{ND}(120)$ & ND (110) & - \\
\hline
\end{tabular}

${ }^{a}$ Fitted $\mathrm{pEC}_{50}$ values are shown to two significant figures when potentiation at $30 \mu \mathrm{M}$ exceeded $130 \%$; values in brackets are the $95 \%$ confidence interval for the corresponding log fitted $\mathrm{pEC}_{50}$ 
value and values in parentheses are the fitted maximum response as a percentage of the initial glutamate $(100 \mu \mathrm{M})$ and glycine $(30 \mu \mathrm{M})$ current. Data are from between 6 and 19 oocytes from 2-3 frogs for each compound and receptor. ${ }^{b}$ Previously published data for CIQ, 188, 187, ${ }^{*}, 198$, 197, and 100 were included for comparison. ${ }^{1}{ }^{c}$ Compound $1 *$ is compound 1 from Santangelo et $a l .^{1}$

Table S3 demonstrates SAR is transferable between the CIQ scaffold and the dihydroisoquinolinone scaffold. Compounds S1-S6 were synthesized according to Scheme S2. The activity patterns are maintained upon derivatization of the A-ring while the remainder of the molecule stays equivalent. For example, on the CIQ scaffold, there is a loss of activity comparing CIQ to $\mathbf{1 8 8}, \mathbf{1 8 7}$, and $\mathbf{1 *}$, there is an improvement in activity comparing CIQ to 198, and activity is maintained comparing CIQ to 197. Similarly, on the dihydroisoquinolinone scaffold, there is a loss of activity comparing $\mathbf{1 2 e}$ to $\mathbf{S 1}, \mathbf{S 2}$, and $\mathbf{S 3}$, there is an improvement in activity comparing 12e to S4, and activity is maintained comparing $12 \mathrm{e}$ to S5. This also applies to derivatization of the B-ring while the remainder of the molecule stays equivalent. For example, there is a loss of activity comparing CIQ to $\mathbf{1 0 0}$ and similarly on the dihydroisoquinolinone scaffold, comparing S5 to S6. 
Supplemental Table S5. Off-Target Actions of Compounds 25i, 31a, and EU-1180-453 at Ligand-Gated Ion Channels Expressed in Xenopus Oocytes ${ }^{c}$

\begin{tabular}{|c|c|c|c|c|}
\hline Receptor & Agonist & $\begin{array}{c}\% \text { control } \\
10 \mu \mathrm{M} \mathrm{compound} \\
31 \mathrm{a}^{a}\end{array}$ & $\begin{array}{c}\% \text { control } \\
10 \mu \mathrm{M} \mathrm{compound} \\
25 \mathrm{i}^{a}\end{array}$ & $\begin{array}{c}\text { \% control } \\
10 \mu M \text { EU-1180- } \\
453^{a, b}\end{array}$ \\
\hline GluN1/GluN2A & $\begin{array}{c}100 \mu \mathrm{M} \text { glutamate, } \\
30 \mu \mathrm{M} \text { glycine }\end{array}$ & $91 \pm 2.6(5)^{b}$ & $97 \pm 1.9(7)^{b}$ & $100 \pm 1.1(8)^{b}$ \\
\hline GluN1/GluN2B & $\begin{array}{c}100 \mu \mathrm{M} \text { glutamate } \\
30 \mu \mathrm{M} \text { glycine }\end{array}$ & $89 \pm 3.4(8)^{b}$ & $99 \pm 4.2(7)^{b}$ & $100 \pm 2.0(8)^{b}$ \\
\hline GluN1/GluN2C & $\begin{array}{c}100 \mu \mathrm{M} \text { glutamate, } \\
30 \mu \mathrm{M} \text { glycine }\end{array}$ & $270 \pm 27(9)^{b}$ & $350 \pm 20(12)^{b}$ & $320 \pm 17(12)^{b}$ \\
\hline GluN1/GluN2D & $\begin{array}{c}100 \mu \mathrm{M} \text { glutamate } \\
30 \mu \mathrm{M} \text { glycine }\end{array}$ & $380 \pm 17(16)^{b}$ & $380 \pm 15(18)^{b}$ & $340 \pm 17(18)^{b}$ \\
\hline GluA1 & $100 \mu \mathrm{M}$ glutamate & $94 \pm 8.7(4)$ & $100 \pm 7.7(5)$ & $99 \pm 1.6(8)$ \\
\hline GluA2-R607Q & $100 \mu \mathrm{M}$ glutamate & $99 \pm 4.8(3)$ & $100 \pm 1.8(4)$ & $100 \pm 1.2(6)$ \\
\hline hGluA3-L531Y & $100 \mu \mathrm{M}$ glutamate & $97 \pm 1.2(10)$ & $97 \pm 1.2(10)$ & $95 \pm 2.5(7)$ \\
\hline hGluA4-L505Y & $100 \mu \mathrm{M}$ glutamate & $99 \pm 0.38(6)$ & $98 \pm 0.65(6)$ & $94 \pm 6.8(5)$ \\
\hline GluK2 & $100 \mu \mathrm{M}$ glutamate & $57 \pm 4.2(5)$ & $110 \pm 17(6)$ & $80 \pm 14(4)$ \\
\hline hGluN1/GluN3A ${ }^{c}$ & $100 \mu \mathrm{M}$ glycine & $100 \pm 0.75(8)$ & $88 \pm 3.0(10)$ & $96 \pm 1.0(10)$ \\
\hline hGluN1/GluN3B ${ }^{c}$ & $100 \mu \mathrm{M}$ glycine & $74 \pm 1.5(12)$ & $85 \pm 2.6(7)$ & $65 \pm 1.9(8)$ \\
\hline$\alpha 1 \beta 2 \gamma \delta$-nACh & $1 \mu \mathrm{M}$ acetylcholine & $66 \pm 2.6(6)$ & $64 \pm 2.8(6)$ & $61 \pm 5.3(7)$ \\
\hline$\alpha 4 \beta 2-n A C h$ & $10 \mu \mathrm{M}$ acetylcholine & $32 \pm 5.8(6)$ & $61 \pm 6.9(8)$ & $60 \pm 5.5(8)$ \\
\hline$\alpha 7-\mathrm{nACh}$ & $300 \mu \mathrm{M}$ acetylcholine & $130 \pm 8.9(5)$ & $100 \pm 5.8(8)$ & $69 \pm 12(6)$ \\
\hline $5-\mathrm{HT}_{3 \mathrm{~A}}$ & $100 \mu \mathrm{M}$ serotonin & $76 \pm 7.6(7)$ & $98 \pm 6.3(7)$ & $83 \pm 2.9(8)$ \\
\hline$\alpha 1 \beta 2 \gamma 2 S-G A B A_{A}$ & $100 \mu \mathrm{M}$ GABA & $94 \pm 2.8(8)$ & $89 \pm 2.3(12)$ & $89 \pm 2.3(8)$ \\
\hline$\rho-\mathbf{G A B A}_{C}$ & $100 \mu \mathrm{M}$ GABA & $120 \pm 6.6(8)$ & $98 \pm 3.5(8)$ & $98 \pm 2.0(8)$ \\
\hline$\alpha 1-G l y c i n e$ & $100 \mu \mathrm{M}$ glycine & $76 \pm 2.2(8)$ & $78 \pm 4.9(5)$ & $67 \pm 1.6(3)$ \\
\hline hP2x & $9 \mu \mathrm{M}$ АТP & $72 \pm 8.0(6)$ & $100 \pm 1.4(4)$ & $85 \pm 2.1(5)$ \\
\hline
\end{tabular}

${ }^{a}$ The mean \pm standard error of the mean for agonist plus $10 \mu \mathrm{M}$ 25i, 31a, or EU-1180-453 as a percent of agonist in vehicle are given for each compound and receptor tested to two significant figures. ${ }^{b}$ Percent control at $30 \mu \mathrm{M}$ test compound for all NMDARs. ${ }^{c} \mathrm{GluN} 1$ contained two mutations (GluN1-F484A,T518L) that removed a GluN1-dependent inhibition of the response. Number of oocytes tested is given in parentheses is number of oocytes. ND $=$ not determined. 
Supplemental Table S6. Off-Target Actions of Compounds 31a, 25i, and EU-1180-453 ${ }^{a}$

\begin{tabular}{|c|c|c|c|c|c|c|}
\hline Receptor & $\begin{array}{c}\text { Radioligand } \\
\text { binding in } \\
10 \mu \mathrm{M} \\
\text { compound } \\
\text { 31a } \\
\text { (\% control) } \\
\end{array}$ & $\begin{array}{c}\mathbf{p K}_{\mathrm{i}} \text { values from } \\
\text { radioligand } \\
\text { competition } \\
\text { binding } \\
\text { isotherms } \\
\text { (compound 31a) }\end{array}$ & $\begin{array}{c}\text { Radioligand } \\
\text { binding in } \\
10 \mu \mathrm{M} \\
\text { compound } \\
25 \mathrm{i} \\
(\% \text { control) } \\
\end{array}$ & $\begin{array}{c}\text { pK }_{\mathrm{i}} \text { values from } \\
\text { radioligand } \\
\text { competition } \\
\text { binding } \\
\text { isotherm } \\
\text { (compound 25i) } \\
\end{array}$ & $\begin{array}{c}\text { Radioligand } \\
\text { binding in } \\
10 \mu \mathrm{M} \mathrm{EU}- \\
1180-453 \\
(\% \text { control) }\end{array}$ & $\begin{array}{c}\mathbf{p K}_{\mathrm{i}} \text { values from } \\
\text { radioligand } \\
\text { competition } \\
\text { binding } \\
\text { isotherm (EU- } \\
\mathbf{1 1 8 0 - 4 5 3 )} \\
\end{array}$ \\
\hline $5-\mathrm{HT}_{1 \mathrm{~A}}$ & 101 & - & 110 & - & 100 & - \\
\hline $5-\mathrm{HT}_{1 \mathrm{~B}}$ & 110 & - & 95 & - & 99 & - \\
\hline 5-HT $1 \mathrm{D}$ & 120 & - & 110 & - & 81 & - \\
\hline $5-\mathrm{HT}_{1 \mathrm{E}}$ & 80 & - & 78 & - & 100 & - \\
\hline $5-\mathrm{HT}_{2 \mathrm{~A}}$ & 49 & $5.7 \pm 0.1$ & 69 & - & 87 & - \\
\hline $5-\mathrm{HT}_{2 \mathrm{~B}}$ & 78 & - & 97 & - & 78 & - \\
\hline $5-\mathrm{HT}_{2 \mathrm{C}}$ & 32 & $<5.0$ & 110 & - & 87 & - \\
\hline $5-\mathrm{HT}_{3}$ & 68 & - & 120 & - & 100 & - \\
\hline $5-\mathrm{HT}_{5 \mathrm{~A}}$ & 92 & - & 95 & - & 87 & - \\
\hline $5-\mathrm{HT}_{6}$ & 85 & - & 75 & - & 49 & - \\
\hline $5-\mathrm{HT}_{7}$ & 83 & - & 120 & - & 100 & - \\
\hline $\mathbf{D}_{1}$ & 87 & - & 59 & - & 87 & - \\
\hline $\mathbf{D}_{2}$ & 100 & - & 87 & - & 100 & - \\
\hline $\mathbf{D}_{3}$ & 93 & - & 71 & - & 96 & - \\
\hline $\mathrm{D}_{4}$ & 100 & - & 90 & - & 96 & - \\
\hline$D_{5}$ & 67 & - & 37 & $5.9 \pm 0.2$ & 70 & - \\
\hline $\begin{array}{l}\text { serotonin } \\
\text { transporter }\end{array}$ & 91 & - & 86 & - & 61 & - \\
\hline $\begin{array}{c}\text { norepinephrine } \\
\text { transporter }\end{array}$ & 70 & - & 75 & - & ND & - \\
\hline $\begin{array}{c}\text { dopamine } \\
\text { transporter }\end{array}$ & 76 & - & 98 & - & 101 & - \\
\hline $\begin{array}{l}\mu \text {-opioid } \\
\text { receptor }\end{array}$ & 99 & - & 100 & - & 113 & - \\
\hline $\begin{array}{l}\delta \text {-opioid } \\
\text { receptor }\end{array}$ & 93 & - & 89 & - & 90 & - \\
\hline $\begin{array}{l}\text { к-opioid } \\
\text { receptor }\end{array}$ & 47 & $5.8 \pm 0.1$ & 32 & $5.8 \pm 0.1$ & 48 & $5.4 \pm 0.1$ \\
\hline GABAA & 120 & - & 110 & - & ND & - \\
\hline $\mathrm{H}_{1}$ & 86 & - & 74 & - & 83 & - \\
\hline $\mathbf{H}_{2}$ & 100 & - & 75 & - & ND & - \\
\hline $\mathbf{H}_{3}$ & 79 & - & 99 & - & 88 & - \\
\hline $\mathbf{H}_{4}$ & 110 & - & 90 & - & 110 & - \\
\hline$\alpha_{1 \mathrm{~A}}$ & 97 & - & 91 & - & 110 & - \\
\hline$\alpha_{1 \mathrm{~B}}$ & 100 & - & 100 & - & 110 & - \\
\hline$\alpha_{1 \mathrm{D}}$ & 79 & - & 79 & - & 99 & - \\
\hline$\alpha_{2 \mathrm{~A}}$ & 98 & - & 79 & - & 74 & - \\
\hline$\alpha_{2 B}$ & 94 & - & 93 & - & 101 & - \\
\hline $\boldsymbol{\alpha}_{2 \mathrm{C}}$ & 85 & - & 83 & - & 86 & - \\
\hline$\beta_{1}$ & 80 & - & 98 & - & ND & - \\
\hline$\beta_{3}$ & 73 & - & 74 & - & ND & - \\
\hline $\mathbf{M}_{1}$ & 88 & - & 90 & - & 87 & - \\
\hline $\mathbf{M}_{2}$ & 90 & - & 110 & - & 140 & - \\
\hline $\mathbf{M}_{3}$ & 87 & - & 69 & - & 120 & - \\
\hline $\mathbf{M}_{4}$ & 100 & - & 84 & - & 71 & - \\
\hline $\mathbf{M}_{5}$ & 110 & - & 100 & - & 76 & - \\
\hline
\end{tabular}




\begin{tabular}{|c|c|c|c|c|c|c|}
\hline $\begin{array}{c}\text { benzodiazapine } \\
\text { rat brain site }\end{array}$ & 88 & - & 71 & - & ND & - \\
\hline $\begin{array}{c}\text { translocator } \\
\text { protein } \\
\text { (peripheral } \\
\text { benzodiazepine } \\
\text { site) }\end{array}$ & 84 & - & 67 & - & 71 & - \\
\hline hERG binding & 70 & - & 73 & - & ND & - \\
\hline$\sigma_{1}$ & 76 & - & 76 & - & 110 & - \\
\hline$\sigma_{2}$ & 32 & $5.8 \pm 0.1$ & 48 & $5.8 \pm 0.1$ & 71 & - \\
\hline
\end{tabular}

${ }^{a}$ Receptor binding profiles of compound 25i, 31a, and EU-1180-453 were generously provided by the National Institute of Mental Health's Psychoactive Drug Screening Program, Contract \# HHSN-271-2013-00017-C (NIMH PDSP). The NIMH PDSP is Directed by Bryan L. Roth at the University of North Carolina at Chapel Hill and Project Officer Jamie Driscoll at NIMH, Bethesda MD, USA. Radioligand binding was measured in the presence of $10 \mu \mathrm{M}$ drug. $\mathrm{A} \mathrm{K}_{\mathrm{i}}$ value was determined for each receptor at which compound showed $>50 \%$ inhibition. For experimental details please refer to the PDSP web site https://pdsp.unc.edu/ims/investigator/web/. ND = not determined. 


\section{Crystal Structure Methods:}<smiles>COc1ccc(OC[C@@H]2c3cccn3CC(=O)N2Cc2cccc(F)c2)cc1</smiles>

Single colorless block-shaped crystals of $(+)-\mathbf{E U - 1 1 8 0 - 4 5 3}$ were crystallized from a 3:1 mixture of hexanes:isopropanol $(\sim 4 \mathrm{mg} / \mathrm{mL})$ at room temperature overnight. A suitable crystal $\left(0.40 \times 0.32 \times 0.22 \mathrm{~mm}^{3}\right)$ was selected and mounted on a loop with paratone oil on an XtaLAB Synergy-S diffractometer. The crystal was kept at a steady $T=99.99(17) \mathrm{K}$ during data collection. The structure was solved with the ShelXT (Sheldrick, 2015) structure solution program using the Intrinsic Phasing solution method and by using Olex2 (Dolomanov et al., 2009) as the graphical interface. The model was refined with version 2018/3 of ShelXL (Sheldrick, 2015) using Least Squares minimization. Crystal data: $\mathrm{C}_{22} \mathrm{H}_{21} \mathrm{FN}_{2} \mathrm{O}_{3}, M_{r}=380.41$, monoclinic, $P 2_{1}$ (No. 4), a = 9.5577(5) $\AA, \mathrm{b}=6.4505(3) \AA, \mathrm{c}=15.2496(8) \AA, \alpha=90^{\circ}, \beta=98.508(5)^{\circ}, \gamma=90^{\circ}, V=929.84(8)$ $\AA^{3}, \mathrm{~T}=99.99(17) \mathrm{K}, Z=2, Z=1, \mu\left(\mathrm{CuK}_{\alpha}\right)=0.806 \mathrm{~mm}^{-1}, 9222$ reflections measured, and of these 2879 were unique $\left(R_{\text {int }}=0.0665\right)$ which were used in all calculations. The final $w R_{2}$ was 0.1166 (all data) and $R_{1}$ was $0.0439(\mathrm{I}>2 \sigma(\mathrm{I}))$. Data collected and analyzed by John Bacsa, PhD at the Emory X-crystallography core facility. Crystallographic data is summarized in Table S2. 


\section{Synthesis of Intermediate 61: Scheme and Experimental Details}

Scheme S1. Synthesis of Phenyl-Based Compound 61

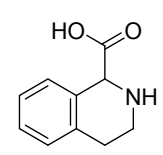

33

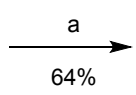

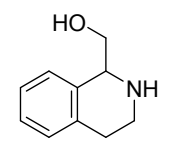

34

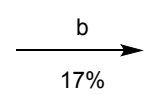

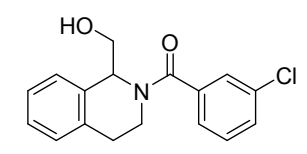

35

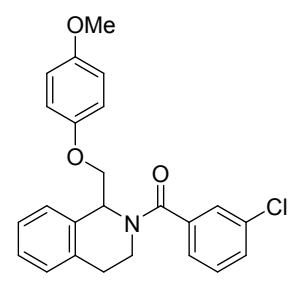

36

${ }^{a}$ Reagents and conditions: (a) $\mathrm{BH}_{3}$-DMS complex, THF, rt, overnight; (b) 3-chlorobenzoyl chloride, $\mathrm{Et}_{3} \mathrm{~N}, \mathrm{DCM}$, rt, 1 h; (c) 4-methoxyiodobenzene, $\mathrm{CuI}, \mathrm{Cs}_{2} \mathrm{CO}_{3}$, 4-Me-Phen, PhMe, 110 ${ }^{\circ} \mathrm{C}, 48 \mathrm{~h}$.

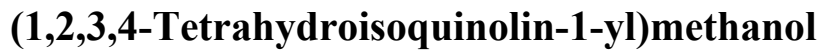

carboxylic acid (33) (1.00 g, $5.64 \mathrm{mmol})$ was dissolved in THF (56 mL) and borane dimethyl sulfide complex $(1.88 \mathrm{~mL}, 19.8 \mathrm{mmol})$ was added dropwise via syringe pump at a rate of $0.1 \mathrm{~mL}$ per minute. The reaction was stirred overnight at room temperature before quenching with $\mathrm{MeOH}$ carefully at room temperature. $1 \mathrm{M} \mathrm{NaOH}$ was added and the organic layer extracted with EtOAc before the combined organic layers were washed once more with $1 \mathrm{M} \mathrm{NaOH}$, washed with brine (3x), dried over $\mathrm{MgSO}_{4}$, and concentrated in vacuo. The crude product was purified via flash column chromatography (ISCO, Redisep $24 \mathrm{~g}$ column, 0-20\% MeOH/DCM gradient) to afford the title compound as a white foam. $(0.59 \mathrm{~g}, 64 \%) . \mathrm{R}_{\mathrm{f}}\left(80: 20: 6 \mathrm{DCM}: \mathrm{MeOH}: \mathrm{NH}_{3}\right): 0.71 ;{ }^{1} \mathrm{H}$ NMR (399 MHz, CDCl3) $\delta 7.19-7.04(\mathrm{~m}, 4 \mathrm{H}), 4.04(\mathrm{dd}, \mathrm{J}=9.3,4.3 \mathrm{~Hz}, 1 \mathrm{H}), 3.79(\mathrm{dd}, \mathrm{J}=10.9,4.3$ $\mathrm{Hz}, 1 \mathrm{H}), 3.65(\mathrm{dd}, \mathrm{J}=10.9,9.3 \mathrm{~Hz}, 1 \mathrm{H}), 3.17-2.97(\mathrm{~m}, 2 \mathrm{H}), 2.81-2.68(\mathrm{~m}, 4 \mathrm{H}) .{ }^{13} \mathrm{C}$ NMR $(100$ $\mathrm{MHz}, \mathrm{CDCl} 3) \delta 135.5,135.2,129.4,126.5,126.2,126.0,64.1,56.4,38.9,29.6$. HRMS calcd. for $\mathrm{C}_{10} \mathrm{H}_{14} \mathrm{ON}, 164.10699[\mathrm{M}+\mathrm{H}]^{+}$; found 164.10683 [M+ H] $]^{+}$. 
General procedure IX was followed using compound $34(0.59 \mathrm{~g}, 3.6 \mathrm{mmol})$, triethylamine (1.00 $\mathrm{mL}, 7.23 \mathrm{mmol})$ and 3-chlorobenzoyl chloride $(0.46 \mathrm{~mL}, 3.6 \mathrm{mmol})$ in DCM $(56 \mathrm{~mL})$. The crude product was purified via flash column chromatography (ISCO, Redisep $24 \mathrm{~g}$ column, 0-100\% EtOAc/hexanes gradient) to afford the title compound as a mixture of rotamers in solution and a clear foam. (0.18 g, 17\%). $\mathrm{R}_{\mathrm{f}}\left(90: 10: 2 \mathrm{DCM}: \mathrm{MeOH}: \mathrm{NH}_{3}\right): 0.55 ;{ }^{1} \mathrm{H}$ NMR $(399 \mathrm{MHz}, \mathrm{CDCl} 3) \delta$ $7.51-7.11(\mathrm{~m}, 8 \mathrm{H}), 6.92(\mathrm{~d}, \mathrm{~J}=7.5 \mathrm{~Hz}, 0.2 \mathrm{H}), 5.84(\mathrm{dd}, \mathrm{J}=8.8,3.9 \mathrm{~Hz}, 0.8 \mathrm{H}), 4.90(\mathrm{dd}, \mathrm{J}=8.7$, $3.9 \mathrm{~Hz}, 0.2 \mathrm{H}), 4.82(\mathrm{dd}, \mathrm{J}=13.1,5.8 \mathrm{~Hz}, 0.2 \mathrm{H}), 4.13-4.06(\mathrm{~m}, 0.8 \mathrm{H}), 3.95(\mathrm{dd}, \mathrm{J}=11.5,9.0 \mathrm{~Hz}$ $0.8 \mathrm{H}), 3.89-3.75(\mathrm{~m}, 1 \mathrm{H}), 3.74-3.54(\mathrm{~m}, 1 \mathrm{H}), 3.35-3.21(\mathrm{~m}, 0.2 \mathrm{H}), 3.20-3.07(\mathrm{~m}, 0.2 \mathrm{H})$, $3.07-2.93(\mathrm{~m}, 0.8 \mathrm{H}), 2.89-2.74(\mathrm{~m}, 1.8 \mathrm{H}) .{ }^{13} \mathrm{C} \mathrm{NMR}\left(151 \mathrm{MHz}, \mathrm{CDCl} 3\right.$, probe temp: $\left.-25^{\circ} \mathrm{C}\right) \delta$ $171.6,170.8,137.6,137.2,134.7,134.5,134.3,133.7,132.3,132.3,130.4,130.3,129.9,129.8$, $129.5,129.2,127.9,127.6,127.6,127.4,127.0,126.8,126.3,125.9,125.0,66.5,64.4,60.1,55.5$ 41.8, 35.2, 29.2, 28.2. HRMS calcd. for $\mathrm{C}_{17} \mathrm{H}_{17} \mathrm{O}_{2} \mathrm{NCl}, 302.09423[\mathrm{M}+\mathrm{H}]^{+}$; found $302.09389[\mathrm{M}$ $+\mathrm{H}]^{+}$.

\section{(3-Chlorophenyl)(1-((4-methoxyphenoxy)methyl)-3,4-dihydroisoquinolin-2(1H)-}

yl)methanone (36). An oven-dried microwave vial was charged with an oven-dried stir bar, compound 35 (100 mg, $0.331 \mathrm{mmol}, 1 \mathrm{eq}$ ), copper(I) iodide (12 mg, $0.066 \mathrm{mmol}, 0.2 \mathrm{eq}), 3,4,7,8-$ tetramethyl-1,10-phenanthroline (16 mg, $0.066 \mathrm{mmol}, 0.2 \mathrm{eq})$, 1-iodo-4-methoxybenzene (116 $\mathrm{mg}, 0.497 \mathrm{mmol}, 1.5 \mathrm{eq}$ ), and cesium carbonate (162 mg, $0.497 \mathrm{mmol}, 1.5 \mathrm{eq})$. The vessel was then capped and evacuated and back-filled with dry argon 3 times. Dry toluene $(0.66 \mathrm{~mL})$ was then added, the vial evacuated and back-filled with dry argon once more and the reaction stirred at 110 ${ }^{\circ} \mathrm{C}$ for 48 hours. The reaction mixture was then cooled to room temperature, diluted with EtOAc, and filtered through a plug of celite, washing with additional EtOAc. The crude material was then 
concentrated and purified via flash column chromatography (ISCO, Redisep $4 \mathrm{~g}$ column, 0-70\% EtOAc/hexanes gradient) to afford the title compound as rotamers in solution and a white foam. (70 mg, 52\%). $\mathrm{R}_{\mathrm{f}}(1: 1 \mathrm{EtOAc:Hex}): 0.68 ;{ }^{1} \mathrm{H}$ NMR $\left(600 \mathrm{MHz}, \mathrm{CDCl} 3\right.$, probe temp: $\left.-25^{\circ} \mathrm{C}\right) \delta 7.65$ (bs, 0.5H), $7.46-7.17(\mathrm{~m}, 7 \mathrm{H}), 7.04(\mathrm{~d}, \mathrm{~J}=7.6 \mathrm{~Hz}, 0.5 \mathrm{H}), 6.89-6.78(\mathrm{~m}, 4 \mathrm{H}), 6.10-6.05(\mathrm{~m}$, $0.5 \mathrm{H}), 5.25-5.20(\mathrm{~m}, 0.5 \mathrm{H}), 4.90(\mathrm{dd}, \mathrm{J}=13.0,5.7 \mathrm{~Hz}, 0.5 \mathrm{H}), 4.45-4.35(\mathrm{~m}, 1 \mathrm{H}), 4.17(\mathrm{t}, \mathrm{J}=$ $10.2 \mathrm{~Hz}, 0.5 \mathrm{H}), 3.95(\mathrm{dd}, \mathrm{J}=10.1,3.7 \mathrm{~Hz}, 0.5 \mathrm{H}), 3.85-3.79(\mathrm{~m}, 0.5 \mathrm{H}), 3.77(\mathrm{~s}, 1.5 \mathrm{H}), 3.77(\mathrm{~s}$ 1.5H), $3.72(\mathrm{td}, \mathrm{J}=13.5,3.7 \mathrm{~Hz}, 0.5 \mathrm{H}), 3.27(\mathrm{td}, \mathrm{J}=12.8,3.7 \mathrm{~Hz}, 0.5 \mathrm{H}), 3.18(\mathrm{td}, \mathrm{J}=14.6,12.6$, $5.9 \mathrm{~Hz}, 0.5 \mathrm{H}), 2.96(\mathrm{ddd}, \mathrm{J}=17.0,12.1,5.8 \mathrm{~Hz}, 0.5 \mathrm{H}), 2.92-2.87(\mathrm{~m}, 0.5 \mathrm{H}), 2.81(\mathrm{~d}, \mathrm{~J}=16.0$ $\mathrm{Hz}, 0.5 \mathrm{H}) .{ }^{13} \mathrm{C}$ NMR $\left(151 \mathrm{MHz}, \mathrm{CDCl} 3\right.$, probe temp: $\left.-25{ }^{\circ} \mathrm{C}\right) \delta 170.4,169.6,153.8,153.7,152.5$, $152.0,137.8,137.7,135.1,134.6,134.3,134.1,132.7,131.8,129.9,129.8,129.2,128.0,127.4$ $127.4,127.2,126.8,126.8,126.4,125.7,124.7,115.3,114.9,114.5,114.4,70.6,69.3,57.4,55.7$, 51.9, 42.6, 35.1, 29.5, 28.1. HRMS calcd. for $\mathrm{C}_{24} \mathrm{H}_{23} \mathrm{O}_{3} \mathrm{NCl}, 408.13610[\mathrm{M}+\mathrm{H}]^{+}$; found $408.13561[\mathrm{M}+\mathrm{H}]^{+}$. Purity was established using an Agilent pump on a Zorbax XBD-C18 column (4.6 $\mathrm{mm} \times 50 \mathrm{~mm}, 3.5 \mu \mathrm{m}$ ). Method 1: $75-95 \% \mathrm{MeOH}$ in water over $3 \mathrm{~min}$ at $1 \mathrm{~mL} / \mathrm{min}$ (retention time $=1.46 \mathrm{~min}$ ). Method 2: $85-95 \% \mathrm{MeOH}$ in water over $5 \mathrm{~min}$ at $1 \mathrm{~mL} / \mathrm{min}$ (retention time 0.84 $\min )$. 


\section{Synthesis of Compounds S1-S6: Scheme and Experimental Details}

Scheme S2. Synthesis of Compounds S1-S6

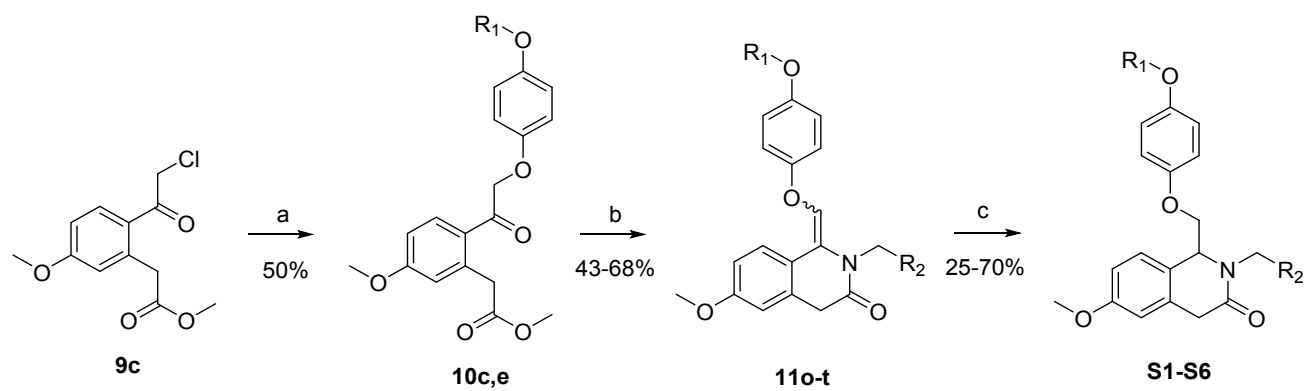

${ }^{a}$ Reagents and conditions: (a) substituted phenol, KI, K2CO3, reflux, 4 h; (b) substituted primary amines, $\mathrm{Ti}(\mathrm{OiPr})_{4}, \mathrm{NaBH}(\mathrm{OAc})_{3}, \mathrm{DCE}, 120{ }^{\circ} \mathrm{C}, \mu \mathrm{w}, 20 \mathrm{~m}$; (c) (i) $\mathrm{PtO}_{2}, \mathrm{H}_{2}$ (balloon), $\mathrm{EtOH}, \mathrm{rt}, 10$ h, (ii) $\mathrm{Pd} / \mathrm{C}, \mathrm{H}_{2}$ (50 psi), EtOH, rt, 24 h.

Methyl 2-(2-(2-(4-ethoxyphenoxy)acetyl)-5-methoxyphenyl)acetate (10e). General procedure II was followed using 9c (500 mg, $1.95 \mathrm{mmol})$, potassium iodide (288 mg, $2.34 \mathrm{mmol})$, 4methoxyphenol (323 mg, $2.34 \mathrm{mmol})$, and potassium carbonate $(538 \mathrm{mg}, 3.90 \mathrm{mmol})$ in acetone $(12 \mathrm{~mL})$. The crude product was purified via flash column chromatography (ISCO, Redisep $12 \mathrm{~g}$ column, 0-50\% EtOAc/hexanes gradient) to afford the title compound as a white solid (352 $\mathrm{mg}$, 50\%). $\mathrm{R}_{\mathrm{f}}(1: 1 \mathrm{EtOAC}: \mathrm{Hex}): 0.74 ;{ }^{1} \mathrm{H} \mathrm{NMR}(500 \mathrm{MHz}, \mathrm{CDCl} 3) \delta 7.84(\mathrm{~d}, \mathrm{~J}=8.7 \mathrm{~Hz}, 1 \mathrm{H}), 6.91-$ $6.77(\mathrm{~m}, 6 \mathrm{H}), 5.10(\mathrm{~s}, 2 \mathrm{H}), 3.96(\mathrm{q}, \mathrm{J}=6.1 \mathrm{~Hz}, 2 \mathrm{H}), 3.95(\mathrm{~s}, 2 \mathrm{H}), 3.86(\mathrm{~s}, 3 \mathrm{H}), 3.67(\mathrm{~s}, 3 \mathrm{H}), 1.37$ $(\mathrm{t}, \mathrm{J}=7.0 \mathrm{~Hz}, 3 \mathrm{H}) .{ }^{13} \mathrm{C} \mathrm{NMR}(126 \mathrm{MHz}, \mathrm{CDCl} 3) \delta 195.8,171.7,162.7,153.7,152.2,138.3,131.8$, 126.9, 119.0, 115.9, 115.4, 112.0, 72.2, 64.0, 55.5, 52.0, 40.4, 14.9. HRMS calcd. for $\mathrm{C}_{20} \mathrm{H}_{23} \mathrm{O}_{6}$, 359.14891 $[\mathrm{M}+\mathrm{H}]^{+}$; found 359.14863 $[\mathrm{M}+\mathrm{H}]^{+}$.

(E/Z)-2-(2-Chlorobenzyl)-6-methoxy-1-((4-methoxyphenoxy)methylene)-1,2-

dihydroisoquinolin-3(4H)-one (110). General procedure III was followed using compound 10c 
(50 mg, $0.15 \mathrm{mmol})$, 2-chlorobenzylamine ( $21 \mu \mathrm{L}, 0.17 \mathrm{mmol})$, tetraisopropoxytitanium $(0.13 \mathrm{~mL}$, $0.44 \mathrm{mmol})$, and sodium triacetoxyborohydride $(92 \mathrm{mg}, 0.44 \mathrm{mmol})$ in DCE $(0.7 \mathrm{~mL})$. The crude product was purified via flash column chromatography (ISCO, Redisep $4 \mathrm{~g}$ column, 0-30\% EtOAc/hexanes gradient) to afford the title compound as a mixture of $E / Z$ isomers and an orange oil (39 mg, 62\%). HRMS calcd. for $\mathrm{C}_{25} \mathrm{H}_{23} \mathrm{O}_{4} \mathrm{NCl}, 436.13101[\mathrm{M}+\mathrm{H}]^{+}$; found $436.13005[\mathrm{M}+$ $\mathrm{H}]^{+}$.

\section{(E/Z)-2-(4-Chlorobenzyl)-6-methoxy-1-((4-methoxyphenoxy)methylene)-1,2-}

dihydroisoquinolin-3(4H)-one (11p). General procedure III was followed using compound 10c (50 mg, $0.15 \mathrm{mmol})$, 4-chlorobezylamine $(21 \mu \mathrm{L}, 0.17 \mathrm{mmol})$, tetraisopropoxytitanium $(0.13 \mathrm{~mL}$, $0.44 \mathrm{mmol})$, and sodium triacetoxyborohydride $(92 \mathrm{mg}, 0.44 \mathrm{mmol})$ in DCE $(0.7 \mathrm{~mL})$. The crude product was purified via flash column chromatography (ISCO, Redisep $4 \mathrm{~g}$ column, 0-30\% EtOAc/hexanes gradient) to afford the title compound as a mixture of $E / Z$ isomers and an orange oil (38 mg, 60\%). HRMS calcd. for $\mathrm{C}_{25} \mathrm{H}_{23} \mathrm{O}_{4} \mathrm{NCl}, 436.13101[\mathrm{M}+\mathrm{H}]^{+}$; found $436.13013[\mathrm{M}+$ $\mathrm{H}]^{+}$.

(E/Z)-2-Benzyl-6-methoxy-1-((4-methoxyphenoxy)methylene)-1,4-dihydroisoquinolin-

3(2H)-one (11q). General procedure III was followed using compound 10c (198 mg, $0.575 \mathrm{mmol})$, benzylamine ( $78 \mu \mathrm{L}, 0.69 \mathrm{mmol})$, tetraisopropoxytitanium $(523 \mu \mathrm{L}, 1.73 \mathrm{mmol})$, and sodium triacetoxyborohydride (366 mg, $1.73 \mathrm{mmol})$ in DCE $(2.9 \mathrm{~mL})$. The crude product was purified via flash column chromatography (ISCO, Redisep $12 \mathrm{~g}$ column, 0-30\% EtOAc/hexanes gradient) to afford the title compound as a mixture of $\mathrm{E} / \mathrm{Z}$ isomers and an orange oil $(0.10 \mathrm{~g}, 43 \%)$. HRMS calcd. for $\mathrm{C}_{25} \mathrm{H}_{24} \mathrm{O}_{4} \mathrm{~N}, 402.16998[\mathrm{M}+\mathrm{H}]^{+}$; found $402.17019[\mathrm{M}+\mathrm{H}]^{+}$.

(E/Z)-2-(3-Fluorobenzyl)-6-methoxy-1-((4-methoxyphenoxy)methylene)-1,4-

dihydroisoquinolin-3(2H)-one (11r). General procedure III was followed using compound 10c 
(300 mg, $0.871 \mathrm{mmol}), 3$-fluorobenzylamine (119 $\mu \mathrm{L}, 1.05 \mathrm{mmol})$, tetraisopropoxytitanium (793 $\mu \mathrm{L}, 2.61 \mathrm{mmol})$, and sodium triacetoxyborohydride $(554 \mathrm{mg}, 2.61 \mathrm{mmol})$ in DCE $(4.4 \mathrm{~mL})$. The crude product was purified via flash column chromatography (ISCO, Redisep $12 \mathrm{~g}$ column, $0-30 \%$ EtOAc/hexanes gradient) to afford the title compound as a mixture of $E / Z$ isomers and an orange oil (250 mg, 68\%). HRMS calcd. for $\mathrm{C}_{25} \mathrm{H}_{23} \mathrm{O}_{4} \mathrm{NF}, 420.16056[\mathrm{M}+\mathrm{H}]^{+}$; found $420.15970[\mathrm{M}+$ $\mathrm{H}]^{+}$.

\section{(E/Z)-6-Methoxy-1-((4-methoxyphenoxy)methylene)-2-(3-(trifluoromethyl)benzyl)-1,4-}

dihydroisoquinolin-3(2H)-one (11s). General procedure III was followed using compound 10c (75 mg, $0.22 \mathrm{mmol}$ ), 3-trifluoromethylbenzylamine (37 $\mu \mathrm{L}, 0.26 \mathrm{mmol})$, tetraisopropoxytitanium (198 $\mu \mathrm{L}, 0.653 \mathrm{mmol})$, and sodium triacetoxyborohydride $(138 \mathrm{mg}, 0.653 \mathrm{mmol})$ in DCE (1.1 $\mathrm{mL}$ ). The crude product was purified via flash column chromatography (ISCO, Redisep $12 \mathrm{~g}$ column, $0-35 \% \mathrm{EtOAc} /$ hexanes gradient) to afford the title compound as a mixture of $\mathrm{E} / \mathrm{Z}$ isomers and a yellow oil (58 mg, 57\%). HRMS calcd. for $\mathrm{C}_{26} \mathrm{H}_{23} \mathrm{O}_{4} \mathrm{NF}_{3}, 470.15737[\mathrm{M}+\mathrm{H}]^{+}$; found $470.15782[\mathrm{M}+\mathrm{H}]^{+}$.

\section{1-((4-Ethoxyphenoxy)methylene)-2-(3-fluorobenzyl)-6-methoxy-1,4-dihydroisoquinolin-}

3(2H)-one (11t). General procedure III was followed using compound 10e (75 $\mathrm{mg}, 0.21 \mathrm{mmol})$, 3-fluorobenzylamine (28 $\mu \mathrm{L}, 0.25 \mathrm{mmol})$, tetraisopropoxytitanium $(0.19 \mathrm{~mL}, 0.63 \mathrm{mmol})$, and sodium triacetoxyborohydride $(0.13 \mathrm{~g}, 0.63 \mathrm{mmol})$ in DCE $(1.0 \mathrm{~mL})$. The crude product was purified via flash column chromatography (ISCO, Redisep $12 \mathrm{~g}$ column, 0-35\% EtOAc/hexanes gradient) to afford the title compound as a mixture of E/Z isomers and a orange oil (40 mg, 44\%).

HRMS calcd. for $\mathrm{C}_{26} \mathrm{H}_{25} \mathrm{O}_{4} \mathrm{NF}, 434.17621[\mathrm{M}+\mathrm{H}]^{+}$; found $434.17626[\mathrm{M}+\mathrm{H}]^{+}$.

\section{2-(2-Chlorobenzyl)-6-methoxy-1-((4-methoxyphenoxy)methyl)-1,2-dihydroisoquinolin-}

3(4H)-one (S1). General procedure IV-A was followed using compound 110 (105 mg, 0.241 
mmol) and platinum(IV) oxide $(7.8 \mathrm{mg}, 0.034 \mathrm{mmol})$ in ethanol $(2 \mathrm{~mL})$. The crude product was purified via flash column chromatography (ISCO, Redisep $4 \mathrm{~g}$ column, 0-30\% EtOAc/hexanes gradient) to afford the title compound as a clear oil. (49 mg, 46\%). $\mathrm{R}_{\mathrm{f}}(1: 1 \mathrm{EtOAc}: \mathrm{Hex}): 0.65 ;{ }^{1} \mathrm{H}$ NMR (400 MHz, CDCl3) $\delta 7.35(\mathrm{~d}, \mathrm{~J}=7.7 \mathrm{~Hz}, 1 \mathrm{H}), 7.20-7.13(\mathrm{~m}, 1 \mathrm{H}), 7.13-7.06(\mathrm{~m}, 3 \mathrm{H})$, $6.83-6.68(\mathrm{~m}, 6 \mathrm{H}), 5.45(\mathrm{~d}, \mathrm{~J}=16.2 \mathrm{~Hz}, 1 \mathrm{H}), 4.71-4.62(\mathrm{~m}, 2 \mathrm{H}), 4.15-4.04(\mathrm{~m}, 2 \mathrm{H}), 3.98(\mathrm{~d}$, $\mathrm{J}=19.2 \mathrm{~Hz}, 1 \mathrm{H}), 3.81(\mathrm{~s}, 3 \mathrm{H}), 3.74(\mathrm{~s}, 3 \mathrm{H}), 3.68(\mathrm{~d}, \mathrm{~J}=19.2 \mathrm{~Hz}, 1 \mathrm{H}) .{ }^{13} \mathrm{C} \mathrm{NMR}(101 \mathrm{MHz}, \mathrm{CDCl} 3)$ $\delta 169.9,159.4,154.2,152.3,134.3,133.3,129.5,128.5,128.4,127.1,127.1,124.7,115.4,114.6$, $112.8,112.3,71.4,60.5,55.7,55.4,46.7,37.7$. HRMS calcd. for $\mathrm{C}_{25} \mathrm{H}_{25} \mathrm{O}_{4} \mathrm{NCl}, 438.14666[\mathrm{M}+$ $\mathrm{H}]^{+}$; found $438.14638[\mathrm{M}+\mathrm{H}]^{+}$. Purity was established using an Agilent pump on a Zorbax XBDC18 column $(4.6 \mathrm{~mm} \times 50 \mathrm{~mm}, 3.5 \mu \mathrm{m})$. Method 1: $85-95 \% \mathrm{MeOH}$ in water over $5 \mathrm{~min}$ at 1 $\mathrm{mL} / \mathrm{min}($ retention time $=1.98 \mathrm{~min}$ ). Method 2: $85-95 \% \mathrm{ACN}$ in water over $6 \mathrm{~min}$ at $1 \mathrm{~mL} / \mathrm{min}$ (retention time $2.11 \mathrm{~min}$ ).

\section{2-(4-Chlorobenzyl)-6-methoxy-1-((4-methoxyphenoxy)methyl)-1,2-dihydroisoquinolin-}

$\mathbf{3 ( 4 H )}$-one (S2). General procedure IV-A was followed using compound $\mathbf{1 1 p}(38 \mathrm{mg}, 0.087 \mathrm{mmol}$ ) and platinum(IV) oxide $(6.6 \mathrm{mg}, 0.029 \mathrm{mmol})$ in ethanol $(5 \mathrm{~mL})$. The crude product was purified via flash column chromatography (ISCO, Redisep $4 \mathrm{~g}$ column, 0-30\% EtOAc/hexanes gradient) to afford the title compound as a clear oil. (18 mg, 47\%). Rf (1:1 EtOAc:Hex): 0.47; ${ }^{1} \mathrm{H}$ NMR (400 MHz, CDCl3) $\delta 7.22(\mathrm{~d}, \mathrm{~J}=8.4 \mathrm{~Hz}, 2 \mathrm{H}), 7.13(\mathrm{~d}, \mathrm{~J}=8.3 \mathrm{~Hz}, 2 \mathrm{H}), 7.05(\mathrm{~d}, \mathrm{~J}=8.4 \mathrm{~Hz}, 1 \mathrm{H}), 6.82-$ $6.67(\mathrm{~m}, 6 \mathrm{H}), 5.46(\mathrm{~d}, \mathrm{~J}=15.4 \mathrm{~Hz}, 1 \mathrm{H}), 4.60(\mathrm{t}, \mathrm{J}=5.1 \mathrm{~Hz}, 1 \mathrm{H}), 4.35(\mathrm{~d}, \mathrm{~J}=15.4 \mathrm{~Hz}, 1 \mathrm{H}), 4.09-$ $3.97(\mathrm{~m}, 2 \mathrm{H}), 3.93(\mathrm{~d}, \mathrm{~J}=19.2 \mathrm{~Hz}, 1 \mathrm{H}), 3.80(\mathrm{~s}, 3 \mathrm{H}), 3.75(\mathrm{~s}, 3 \mathrm{H}), 3.66(\mathrm{~d}, \mathrm{~J}=19.2 \mathrm{~Hz}, 1 \mathrm{H}) .{ }^{13} \mathrm{C}$ NMR (101 MHz, CDCl3) $\delta$ 169.6, 159.4, 154.2, 152.2, 135.5, 134.1, 133.1, 129.1, 128.8, 127.1, 124.6, 115.4, 114.6, 112.8, 112.3, 71.5, 59.7, 55.7, 55.3, 48.4, 37.7. HRMS calcd. for $\mathrm{C}_{25} \mathrm{H}_{25} \mathrm{O}_{4} \mathrm{NCl}, 438.14666[\mathrm{M}+\mathrm{H}]^{+}$; found $438.14656[\mathrm{M}+\mathrm{H}]^{+}$. Purity was established using an 
Agilent pump on a Zorbax XBD-C18 column (4.6 mm $\times 50 \mathrm{~mm}, 3.5 \mu \mathrm{m})$. Method 1: 85-95\% $\mathrm{MeOH}$ in water over $5 \mathrm{~min}$ at $1 \mathrm{~mL} / \mathrm{min}($ retention time $=1.87 \mathrm{~min})$. Method 2: $85-95 \% \mathrm{ACN}$ in water over $6 \mathrm{~min}$ at $1 \mathrm{~mL} / \mathrm{min}$ (retention time $2.02 \mathrm{~min}$ ).

\section{2-Benzyl-6-methoxy-1-((4-methoxyphenoxy)methyl)-1,4-dihydroisoquinolin-3(2H)-one (S3).}

General procedure IV-B was followed using compound $11 q(50 \mathrm{mg}, 0.13 \mathrm{mmol})$ and $10 \% \mathrm{Pd} / \mathrm{C}$ (20 $\mathrm{mg}, 0.019 \mathrm{mmol})$ in ethanol $(5 \mathrm{~mL})$. The crude product was purified via flash column chromatography (ISCO, Redisep $4 \mathrm{~g}$ column, 0-50\% EtOAc/hexanes gradient) to afford the title compound as a clear oil. (14 mg, 28\%). $\mathrm{R}_{\mathrm{f}}(1: 1 \mathrm{EtOAc:Hex}): 0.45 ;{ }^{1} \mathrm{H}$ NMR $(500 \mathrm{MHz}, \mathrm{CDCl} 3) \delta$ $7.28-7.17(\mathrm{~m}, 5 \mathrm{H}), 7.03(\mathrm{~d}, \mathrm{~J}=8.4 \mathrm{~Hz}, 1 \mathrm{H}), 6.80-6.68(\mathrm{~m}, 6 \mathrm{H}), 5.52(\mathrm{~d}, \mathrm{~J}=15.3 \mathrm{~Hz}, 1 \mathrm{H}), 4.62$ (t, J = 5.0 Hz, 1H), $4.37(\mathrm{~d}, \mathrm{~J}=15.3 \mathrm{~Hz}, 1 \mathrm{H}), 4.07-3.98(\mathrm{~m}, 2 \mathrm{H}), 3.94(\mathrm{~d}, \mathrm{~J}=19.2 \mathrm{~Hz}, 1 \mathrm{H}), 3.79$ (s, 3H), $3.74(\mathrm{~s}, 3 \mathrm{H}), 3.67$ (d, J = 19.3 Hz, 1H). ${ }^{13} \mathrm{C}$ NMR (126 MHz, CDCl3) $\delta$ 169.6, 159.4, $154.2,152.3,136.9,134.3,128.7,127.7,127.4,127.1,124.9,115.5,114.7,112.7,112.3,71.5$, 59.6, 55.7, 55.4, 48.9, 37.7. HRMS calcd. for $\mathrm{C}_{25} \mathrm{H}_{26} \mathrm{O}_{4} \mathrm{~N}, 404.18563[\mathrm{M}+\mathrm{H}]^{+}$; found 404.18552 $[\mathrm{M}+\mathrm{H}]^{+}$. Purity was established using an Agilent pump on a Zorbax XBD-C18 column $(4.6 \mathrm{~mm}$ $\times 50 \mathrm{~mm}, 3.5 \mu \mathrm{m})$. Method 1: $85-95 \% \mathrm{MeOH}$ in water over $5 \mathrm{~min}$ at $1 \mathrm{~mL} / \mathrm{min}$ (retention time $=$ $3.66 \mathrm{~min}$ ). Method 2: $85-95 \% \mathrm{ACN}$ in water over $6 \mathrm{~min}$ at $1 \mathrm{~mL} / \mathrm{min}$ (retention time $2.29 \mathrm{~min}$ ). 2-(3-Fluorobenzyl)-6-methoxy-1-((4-methoxyphenoxy)methyl)-1,4-dihydroisoquinolin3(2H)-one (S4). General procedure IV-B was followed using compound $\mathbf{1 1 r}$ (44 mg, $0.11 \mathrm{mmol})$ and $10 \% \mathrm{Pd} / \mathrm{C}(17 \mathrm{mg}, 0.016 \mathrm{mmol})$ in ethanol $(5 \mathrm{~mL})$. The crude product was purified via flash column chromatography (ISCO, Redisep $4 \mathrm{~g}$ column, 0-50\% EtOAc/hexanes gradient) to afford the title compound as a clear oil. (11 mg, $25 \%) . \mathrm{R}_{\mathrm{f}}(1: 1 \mathrm{EtOAc:Hex}): 0.47 ;{ }^{1} \mathrm{H} \mathrm{NMR}(500 \mathrm{MHz}$, CDCl3) $\delta 7.24-7.19(\mathrm{~m}, 1 \mathrm{H}), 7.06(\mathrm{~d}, \mathrm{~J}=8.4 \mathrm{~Hz}, 1 \mathrm{H}), 6.97(\mathrm{~d}, \mathrm{~J}=7.8 \mathrm{~Hz}, 1 \mathrm{H}), 6.91(\mathrm{t}, \mathrm{J}=10.1$ Hz, 2H), $6.81-6.69(\mathrm{~m}, 6 \mathrm{H}), 5.49(\mathrm{~d}, \mathrm{~J}=15.5 \mathrm{~Hz}, 1 \mathrm{H}), 4.63(\mathrm{t}, \mathrm{J}=5.1 \mathrm{~Hz}, 1 \mathrm{H}), 4.38(\mathrm{~d}, \mathrm{~J}=15.5$ 
Hz, 1H), $4.08-4.00(\mathrm{~m}, 2 \mathrm{H}), 3.94(\mathrm{~d}, \mathrm{~J}=19.2 \mathrm{~Hz}, 1 \mathrm{H}), 3.81(\mathrm{~s}, 3 \mathrm{H}), 3.75(\mathrm{~s}, 3 \mathrm{H}), 3.67(\mathrm{~d}, \mathrm{~J}=$ $19.3 \mathrm{~Hz}, 1 \mathrm{H}) .{ }^{13} \mathrm{C}$ NMR $(126 \mathrm{MHz}, \mathrm{CDCl} 3) \delta 169.6,163.0\left(\mathrm{~d}, \mathrm{~J}=246.5 \mathrm{~Hz},{ }^{1} J\right), 159.5,154.3$, 152.2, $139.7\left(\mathrm{~d}, \mathrm{~J}=7.0 \mathrm{~Hz},{ }^{3} \mathrm{~J}\right), 134.2,130.2\left(\mathrm{~d}, \mathrm{~J}=8.2 \mathrm{~Hz},{ }^{3} \mathrm{~J}\right), 127.1,124.6,123.2(\mathrm{~d}, \mathrm{~J}=2.8 \mathrm{~Hz}$ $\left.{ }^{4} J\right), 115.4,114.7,114.6\left(\mathrm{~d}, \mathrm{~J}=21.9 \mathrm{~Hz},{ }^{2} J\right), 114.3\left(\mathrm{~d}, \mathrm{~J}=21.2 \mathrm{~Hz},{ }^{2} J\right), 112.9,112.4,71.6,59.9$, 55.7, 55.4, 48.6, 37.7. HRMS calcd. for $\mathrm{C}_{25} \mathrm{H}_{25} \mathrm{O}_{4} \mathrm{NF}, 422.17621[\mathrm{M}+\mathrm{H}]^{+}$; found $422.17602[\mathrm{M}$ $+\mathrm{H}]^{+}$. Purity was established using an Agilent pump on a Zorbax XBD-C18 column (4.6 mm $\times$ $50 \mathrm{~mm}, 3.5 \mu \mathrm{m}$ ). Method 1: 85-95\% $\mathrm{MeOH}$ in water over $5 \mathrm{~min}$ at $1 \mathrm{~mL} / \mathrm{min}$ (retention time $=$ $1.90 \mathrm{~min}$ ). Method 2: 85-95\% $\mathrm{ACN}$ in water over $6 \mathrm{~min}$ at $1 \mathrm{~mL} / \mathrm{min}$ (retention time $2.07 \mathrm{~min}$ ).

\section{6-Methoxy-1-((4-methoxyphenoxy)methyl)-2-(3-(trifluoromethyl)benzyl)-1,4-}

dihydroisoquinolin-3(2H)-one (S5). General procedure IV-B was followed using compound 11s (58 $\mathrm{mg}, 0.12 \mathrm{mmol})$ and $10 \% \mathrm{Pd} / \mathrm{C}(20 \mathrm{mg}, 0.019 \mathrm{mmol})$ in ethanol $(5 \mathrm{~mL})$. The crude product was purified via flash column chromatography (ISCO, Redisep $4 \mathrm{~g}$ column, 0-50\% EtOAc/hexanes gradient) to afford the title compound as a clear oil. (41 mg, 70\%). $\mathrm{R}_{\mathrm{f}}(1: 1 \mathrm{EtOAc:Hex}): 0.50 ;{ }^{1} \mathrm{H}$ NMR (500 MHz, CDCl3) $\delta 7.51-7.45(\mathrm{~m}, 2 \mathrm{H}), 7.36(\mathrm{~m}, 2 \mathrm{H}), 7.06(\mathrm{~d}, \mathrm{~J}=8.4 \mathrm{~Hz}, 1 \mathrm{H}), 6.80-$ $6.76(\mathrm{~m}, 3 \mathrm{H}), 6.74-6.69(\mathrm{~m}, 3 \mathrm{H}), 5.52(\mathrm{~d}, \mathrm{~J}=15.5 \mathrm{~Hz}, 1 \mathrm{H}), 4.63(\mathrm{t}, \mathrm{J}=5.6 \mathrm{~Hz}, 1 \mathrm{H}), 4.48(\mathrm{~d}, \mathrm{~J}=$ 15.6 Hz, 1H), $4.09-4.01(\mathrm{~m}, 2 \mathrm{H}), 3.95(\mathrm{~d}, \mathrm{~J}=19.2 \mathrm{~Hz}, 1 \mathrm{H}), 3.80(\mathrm{~s}, 3 \mathrm{H}), 3.75(\mathrm{~s}, 3 \mathrm{H}), 3.68(\mathrm{~d}, \mathrm{~J}$ $=19.2 \mathrm{~Hz}, 1 \mathrm{H}) .{ }^{13} \mathrm{C}$ NMR $(126 \mathrm{MHz}, \mathrm{CDCl} 3) \delta 169.8,159.5,154.3,152.1,138.2,134.1,130.9$ $\left(\mathrm{q}, \mathrm{J}=32.1 \mathrm{~Hz},{ }^{2} J\right), 130.9,129.2,127.1,124.4(8), 124.4(8)\left(\mathrm{q}, \mathrm{J}=3.8 \mathrm{~Hz},{ }^{3} J\right), 124.4(9)(\mathrm{q}, \mathrm{J}=3.8$ $\left.\mathrm{Hz},{ }^{3} \mathrm{~J}\right), 124.0\left(\mathrm{q}, \mathrm{J}=272.3 \mathrm{~Hz},{ }^{1} \mathrm{~J}\right), 115.4,114.7,112.9,112.4,71.7,60.1,55.7,55.4,48.9,37.7$. HRMS calcd. for $\mathrm{C}_{26} \mathrm{H}_{25} \mathrm{O}_{4} \mathrm{NF}_{3}, 472.17302[\mathrm{M}+\mathrm{H}]^{+}$; found $472.17339[\mathrm{M}+\mathrm{H}]^{+}$. Purity was established using an Agilent pump on a Zorbax XBD-C18 column $(4.6 \mathrm{~mm} \times 50 \mathrm{~mm}, 3.5 \mu \mathrm{m})$. Method 1: $85-95 \% \mathrm{MeOH}$ in water over $5 \mathrm{~min}$ at $1 \mathrm{~mL} / \mathrm{min}($ retention time $=2.64 \mathrm{~min})$. Method 2: $95 \% \mathrm{MeOH}$ in water over $3 \mathrm{~min}$ at $1 \mathrm{~mL} / \mathrm{min}$ (retention time $0.93 \mathrm{~min}$ ). 


\section{1-((4-Ethoxyphenoxy)methyl)-2-(3-fluorobenzyl)-6-methoxy-1,4-dihydroisoquinolin-3(2H)-}

one (S6). General procedure IV-B was followed using compound 11t (39 mg, $0.090 \mathrm{mmol}$ ) and $10 \% \mathrm{Pd} / \mathrm{C}(14 \mathrm{mg}, 0.013 \mathrm{mmol})$ in ethanol $(5 \mathrm{~mL})$. The crude product was purified via flash column chromatography (ISCO, Redisep $4 \mathrm{~g}$ column, 0-50\% EtOAc/hexanes gradient) to afford the title compound as a clear oil. (18 mg, 46\%). $\mathrm{R}_{\mathrm{f}}\left(1: 1\right.$ EtOAc:Hex): $0.40 ;{ }^{1} \mathrm{H}$ NMR (500 MHz, CDCl3) $\delta 7.24-7.19(\mathrm{~m}, 1 \mathrm{H}), 7.06(\mathrm{~d}, \mathrm{~J}=8.4 \mathrm{~Hz}, 1 \mathrm{H}), 6.97(\mathrm{~d}, \mathrm{~J}=7.7 \mathrm{~Hz}, 1 \mathrm{H}), 6.94-6.88(\mathrm{~m}$, 2H), $6.81-6.75(\mathrm{~m}, 3 \mathrm{H}), 6.74-6.68(\mathrm{~m}, 3 \mathrm{H}), 5.49(\mathrm{~d}, \mathrm{~J}=15.5 \mathrm{~Hz}, 1 \mathrm{H}), 4.63(\mathrm{t}, \mathrm{J}=5.1 \mathrm{~Hz}, 1 \mathrm{H})$, $4.38(\mathrm{~d}, \mathrm{~J}=15.5 \mathrm{~Hz}, 1 \mathrm{H}), 4.08-4.00(\mathrm{~m}, 2 \mathrm{H}), 3.96(\mathrm{q}, \mathrm{J}=6.9 \mathrm{~Hz}, 2 \mathrm{H}), 3.94(\mathrm{~d}, \mathrm{~J}=19.2 \mathrm{~Hz}, 1 \mathrm{H})$, $3.80(\mathrm{~s}, 3 \mathrm{H}), 3.67(\mathrm{~d}, \mathrm{~J}=19.3 \mathrm{~Hz}, 1 \mathrm{H}), 1.38(\mathrm{t}, \mathrm{J}=7.0 \mathrm{~Hz}, 3 \mathrm{H}) .{ }^{13} \mathrm{C} \mathrm{NMR}(126 \mathrm{MHz}, \mathrm{CDCl} 3) \delta$ 169.6, $163.0\left(\mathrm{~d}, \mathrm{~J}=246.5 \mathrm{~Hz},{ }^{1} J\right), 159.5,153.6,152.2,139.7\left(\mathrm{~d}, \mathrm{~J}=6.9 \mathrm{~Hz},{ }^{3} \mathrm{~J}\right), 134.2,130.2(\mathrm{~d}, \mathrm{~J}$ $\left.=8.2 \mathrm{~Hz},{ }^{3} \mathrm{~J}\right), 127.1,124.6,123.2\left(\mathrm{~d}, \mathrm{~J}=2.7 \mathrm{~Hz},{ }^{4} \mathrm{~J}\right), 115.4,114.6\left(\mathrm{~d}, \mathrm{~J}=21.6 \mathrm{~Hz},{ }^{2} \mathrm{~J}\right), 114.3(\mathrm{~d}, \mathrm{~J}$ $\left.=21.1 \mathrm{~Hz},{ }^{2} J\right), 112.9,112.4,71.6,64.0,56.0,55.4,48.6,37.7,14.9$. HRMS calcd. for $\mathrm{C}_{26} \mathrm{H}_{26} \mathrm{O}_{4} \mathrm{NFNa}, 458.17381[\mathrm{M}+\mathrm{Na}]^{+}$; found 458.17390 [M $\left.+\mathrm{Na}\right]^{+}$. Purity was established using an Agilent pump on a Zorbax XBD-C18 column $(4.6 \mathrm{~mm} \times 50 \mathrm{~mm}, 3.5 \mu \mathrm{m})$. Method 1: 85-95\% $\mathrm{MeOH}$ in water over $5 \mathrm{~min}$ at $1 \mathrm{~mL} / \mathrm{min}$ (retention time $=3.14 \mathrm{~min}$ ). Method 2: $85-95 \% \mathrm{ACN}$ in water over $6 \mathrm{~min}$ at $1 \mathrm{~mL} / \mathrm{min}$ (retention time $2.54 \mathrm{~min}$ ). 


\section{$\underline{\text { References: }}$}

1. Santangelo Freel, R. M.; Ogden, K. K.; Strong, K. L.; Khatri, A.; Chepiga, K. M.; Jensen, H. S.; Traynelis, S. F.; Liotta, D. C., Synthesis and structure activity relationship of tetrahydroisoquinoline-based potentiators of GluN2C and GluN2D containing N-methyl-Daspartate receptors. J. Med. Chem. 2013, 56, 5351-5381. 